\title{
Subtle memory and attentional deficits revealed in an Irish stroke patient sample using domain-specific cognitive tasks
}

\author{
Joseph T. Duffin ${ }^{1}$, D. Ronan Collins ${ }^{2}$, Tara Coughlan², Desmond O'Neill ${ }^{2}$, \\ Richard A. P. Roche ${ }^{1}$, and Sean Commins ${ }^{1}$ \\ ${ }^{1}$ Department of Psychology, National University of Ireland Maynooth, Maynooth, Ireland \\ ${ }^{2}$ Age-Related Health Care/Stroke-Service Adelaide and Meath National Children's Hospital, Tallaght, \\ Dublin, Ireland
}

\begin{abstract}
Stroke disrupts motor, sensory, and cognitive systems in survivors. Unlike in physical impairments, assessment of cognitive function is often inadequate, as no standardized procedure to monitor cognitive recovery post stroke exists. We evaluated a number of novel task-orientated tools designed to assess subtle cognitive deficits (including memory, attention, and executive functioning) in a sample of stroke patients. Although unimpaired on MMSE (Mini-Mental State Examination)-based indices of cognition, memory, and intelligence, stroke survivors were significantly impaired on tasks testing visual attention, spatial/relational processing, and associative memory. We recommend a standardized multidomain cognitive assessment and propose that cognitive deficits post stroke require in-depth assessment to inform patient-orientated rehabilitation.
\end{abstract}

Keywords: Stroke; Cognition; Memory; Attention; Assessment.

\section{INTRODUCTION}

A stroke is a severe form of brain injury in which a blood vessel either becomes blocked, cutting off the supply of oxygen to brain cells, or bursts, killing large numbers of neurons. In Ireland, stroke affects over 10,000 people annually (Irish Heart Foundation National Audit of Stroke Care, 2008), and sufferers are frequently left with a range of disabilities. Forty-eight percent of stroke survivors suffer from residual hemiparesis, $22 \%$ are unable to walk, between 24 and $53 \%$ require assistance with daily activities of living, and 32\% suffer from clinical depression (Irish Heart Foundation Council on Stroke, 2001, report). Indeed, according to the same report, $33 \%$ of survivors also have some form of cognitive difficulty. Internationally, up to two thirds of stroke survivors suffer from cognitive impairments in the areas of memory, language, and decision making (McDonnell, Bryan, Smith, \& Esterman, 2011). Such cognitive impairments correlate with poor rehabilitation outcomes including a reduced capacity for work and independent living (Hofgren, Bjorkdahl, Esbjornsson, \& Sunnerhagen, 2007; Hommel, Miguel, Naegele, Gonnet, \& Jaillard, 2009). Furthermore, there is a high risk of patients developing dementia following a recurrent stroke (Pendlebury \& Rothwell, 2009).

There is little general agreement as to what cognitive tests should be administered post stroke. There are numerous scales that monitor recovery in general, such as the Functional Independence Measure (Keith, Granger, Hamilton, \& Sherwin, 1987) or the Barthel Index (Mahoney \& Barthel, 1965), but these scales do not measure cognitive deficits adequately, if at all. General cognitive deficits can, however, be measured by standard pen-and-paper forms of assessment (for example,

Acknowledgements: Irish Research Council for Humanities and Social Sciences; John and Pat Hume NUI Maynooth scholarship.

Address correspondence to Sean Commins, Department of Psychology, NUI Maynooth, Maynooth, Co. Kildare, Ireland (E-mail: Sean.Commins@nuim.ie). 
the Mini Mental State Examination (MMSE; Folstein, Folstein, \& McHugh, 1975), the Clifton Assessment Procedures for the Elderly (CAPE; Pattie, 1981), and Addenbrooke's Cognitive Exam (Mathuranath, Nestor, Berrios, Rakowicz, \& Hodges, 2000). Such scales are also routinely used in the Irish context. However, these tests often miss more subtle or complex deficits in cognition and fail to tap into the multiple facets of a single cognitive domain. For example, if memory impairments are detected, what type of memory deficit is observed-working memory, verbal memory, spatial memory, visual memory, long-term memory, and so on? While assessments such as the MMSE may identify such verbal impairments, often general cognitive assessments fail to adequately measure functions such as attending to relevant input, abstract problem solving, long-term retrieval, and visuospatial ability. Indeed, the recall and attention MMSE subtests tend to be the least reliable (Olin \& Zelinski, 1991), with low test-retest correlations reported.

Although cognitive deficits are common in stroke survivors (Patel, Coshall, Rudd, \& Wolfe, 2003), there is considerable variability in the deficits observed due primarily to the nature and location of damage, which makes assessment difficult to standardize. Some stroke damage can be quite discrete and confined to specific regions. For example, many deficits arise from damage centered on parietal lobe producing deficits in movement guidance (misreaching or apraxia; see Benton, 1990), impaired attention to specific regions of visual space (contralateral neglect; see Mesulam, 1981), deficits in object recognition when viewed from unusual or rotated angles (Warrington \& Taylor, 1973), and disorders of spatial cognition (mental rotation and spatial translations; Farrell \& Robertson, 2000). More commonly, however, large areas of cortex (including regions beyond the parietal lobes) are affected, leading to a constellation of impairments, which may include memory problems and attention/awareness deficits (see Cicerone et al., 2005). Due to the limitations of some current cognitive assessments (e.g., the MMSE has been reported to be biased towards verbal items (Strauss, Sherman, \& Spreen, 2006), the lack of consensus on what assessments should be used, and the difficulty in trying to standardize such assessments given the heterogeneity of the stroke population, there is a need to assess for multiple cognitive domains (e.g., memory, attention, executive function, etc). In addition, when a particular cognitive deficit is detected, there is a need for a deeper assessment of that particular deficit (e.g., for attention, does the deficit lie with alerting and orientation to a stimulus or the maintenance of attention?). A recent study by McDonnell et al. (2011) sought to do this by comparing stroke patients to matched controls on a number of freely available tests that evaluate five cognitive domains including executive function, memory, working memory, speed of processing, and visuospatial function. The authors suggest that their protocol could be used as the basis for a standardized approach to poststroke assessment.

We concur with the suggestion by McDonnell et al. (2011) and attempt to expand upon their work by examining a number of tests that provide a deeper assessment within specific cognitive domains. We report on a number of tasks that we have developed, which can be used alongside standard neuropsychological tests. These tasks include both paper-based assessment and computer-based tasks that can easily be administered to patients at bedside using a standard laptop procedure. The tasks access a variety of cognitive functions but are designed to provide a deeper assessment and to identify subtle deficits primarily in the domains of attention and memory. Specifically, we aim to probe visual attention, spatial processing, relational and location processing, and episodic/associative memory. Consideration should be given to utilizing such novel task-based assessments if they prove sensitive in detecting differences between stroke patients and matched controls as part of a broader multidomain assessment (such as the one proposed by McDonnell et al., 2011).

\section{METHOD}

\section{Participants}

Participants comprised 16 patients aged 30-88 years (mean 65.4 years) who were between 5 and 60 days post stroke. There were 11 males and 5 females. Twenty-six healthy controls between the ages of 24 and 84 years (mean 56.4 years) were also recruited (10 males and 16 females). All recruitment took place during a two-year period from October 2009 to October 2011 at the Acute Stroke Service of the Adelaide \& Meath Hospital, Tallaght, Dublin, Ireland.

\section{Control selection}

Participants for the control group were recruited primarily via word of mouth, by advertisement, and through approaching community centers, including centers for the elderly. All control participants were matched as closely as possible to the stroke sample, with respect to age, gender, and general demographics. For example, participants were generally 
recruited from the greater Dublin and surrounding areas. These geographical regions also form the primary catchment area for Tallaght hospital. Any participants that had a previous medical condition, such as heart attack or stroke, were excluded from the sample. In addition, any participants that had a history of or were currently suffering from a psychiatric or cognitive illness (e.g., Alzheimer disease, dementia, etc.) were also excluded. To confirm that participants had no cognitive problems, a random subsample $(n=5)$ were given the MMSE. These participants had a mean score of 29.6/30. In addition, we asked a separate subset to perform the Interlocking Finger Test (Moo, Slotnick, Tesoro, Zee, \& Hart, 2003) to ensure that they had no motor problems. All participants $(n=9)$ scored $4 / 4$ on this test. All participants spoke English as their primary language and had normal or corrected-to-normal vision. Twenty-four participants were right handed, and one was left-handed.

\section{Patient selection}

Eligibility criteria for inclusion in the study included: a confirmed diagnosis by a stroke physician of stroke as defined by the International Classification of Diseases (ICD)-10 diagnostic criteria; age over 18; between 5-90 days post stroke, and clinically stable. Both first and recurrent and ischemic/hemorrhagic stroke subtypes were included. Exclusion criteria included: severe hemiparesis affecting the dominant side; severe aphasia; severe cognitive deficits (MMSE $<10$ on screening); severe visual neglect; and patients with multifocal white matter ischemia associated with a transient ischemic attack (TIA).

\section{Procedure}

Patients were recruited by approaching them directly in the Stroke Unit. Each gave written informed consent. Following consent, participants in both the stroke and healthy control groups were then given a battery of paper-based assessment tasks (requiring approximately 50 minutes to complete) and a further battery of computer-based tasks (requiring approximately 1 hour and $30 \mathrm{~min}$ to complete). The administration of the tasks varied for each patient/participant with some requiring multiple sessions for completion.

\section{Tasks administered: Paper based}

Four paper-based tasks were used. The National Adult Reading Task (NART; Nelson, 1982) is a test designed to estimate the level of premorbid intellectual functioning in neuropsychological practice and research. The test comprises 50 phonetically irregular words (e.g., ache, naïve, thyme). Assuming that the participant is familiar with the word, accuracy of pronunciation is used to predict IQ. Due to the words being phonetically irregular, phonetic decoding or guesswork will not provide the correct pronunciation. The test relies on the high correlation between reading ability and intelligence in the normal population. The value for IQ is calculated using an equation that contains variables for age, sex, and education level. Willshire, Kinsella, and Prior (1991) used the equation below for participants between 55 and 69: [Estimated IQ $=123.7-$ 0.8 (NART errors) + 3.8 education -7.4 sex] .

The Rey Auditory Verbal Learning Task (RAVLT; Rey, 1941) is designed to assess immediate memory span, new learning, susceptibility to interference, and recognition memory. The test consists of 15 nouns (List A) read out loud for five consecutive trials. After each trial, the participant is asked to recall as many words as they can remember. After the completion of Trial A5, another list, the interference list (List B), is read to the participant, and recall of List B is probed. This is followed by an immediate request to the participant to recall the words from List A again. After a 20 -minute delay, the participant is requested to recall all the words they can remember from List A (Trial A7).

The Trail Making Task (TMT; Reitan, 1992) is a test used to assess visual attention and task switching, sequencing, mental flexibility, visual search, and motor function, as well as executive control. The test is composed of two parts, A and B. Part A requires a participant to "join the dots" or draw a line linking circled numbers in increasing numerical sequence starting from 1 to 25 . In Part B, the participant is required to "join the dots" in alternating numerical and alphabetical order starting with number " 1 " linked to letter " $A$ " then linked to number " 2 " then to letter " $\mathrm{B}$ " so that they complete the number and letter sequence. The time taken in seconds for each part is measured for all participants, and the difference between the parts (i.e., Trial B minus Trial A) is used as an indicator of flexibility and attention.

The Cognitive Failures Questionnaire (CFQ; Broadbent, Cooper, FitzGerald, \& Parkes, 1982) is a 25 -item self-rating questionnaire. Participants report on failures of perception, memory, and motor function, as well as absentmindedness and slips of action, by using the 5-point Likert scale (i.e., $0=$ never, $1=$ very rarely, $2=$ occasionally, 3 = quite often, $4=$ very often), and the total scores range from 0 to 100 . Items include, 
"Do you bump into people?", "Do you fail to notice something although it is there?", and "Do you forget peoples' names?" Higher scores indicate greater absentmindedness.

\section{Tasks: Computer based}

In addition to the paper-based tests, all participants also executed a number of computer-based tasks. Stimuli for each task were presented using E-Prime on a Dell Latitude D600 Pentium laptop. Accuracy and reaction time were recorded automatically.

\section{Visual search}

The visual search paradigm is a well-established task, which depends on selective visual attention (Treisman \& Gelade, 1980) and requires an intact parietal cortex (Humphreys, Allen, \& Mavritsaki, 2009). We designed a modified version of this task consisting of a conjunction search wherein the target was defined by the conjunction of orientation and color; specifically, the target was a red forward slash (/) among distractors, which were red back-slashes $(\backslash)$, red horizontal and vertical line segments, and blue forward slashes, back-slashes, horizontals, and verticals. Each line segment subtended a visual angle of 1.72 degrees. The task involved two blocks of 60 trials each. Within each block, the target stimulus was present on $50 \%$ of trials. Stimulus arrays were presented on screen until a button-press response was made (i.e., no time limit was imposed), and the arrays were interleaved with a central fixation cross, which was presented for $500 \mathrm{~ms}$. Participants were instructed to press the left mouse button to indicate target presence and the right mouse button for target absence. Arrays always contained eight stimulifour red and four blue-irrespective of target presence or absence (i.e., seven distractors for targetpresent trials, eight distractors for target-absent trials).

\section{Spatial Grid Task}

This task was designed to examine spatial processing and mental rotation as well as testing object-location memory (see Johnsrude, Owen, Crane, Milner, \& Evans, 1999 Murphy, Wynne, O'Rourke, Commins, \& Roche, 2009). Stimuli consisted of eight different objects (a post-box, a tree, a keg, a brick, a bean bag, a lamp, a tripod and a top hat) presented on a $4 \times 4$ grid environment created using Google SketchUp, which allowed for three-dimensional rendering in full color. Two landmark objects (a street light and a well) were always present in the environment. All objects were presented in 1 of the 14 free squares in the grid. 3D realism was achieved through the program's use of depth cues, texture gradients, spatial consistency, and overlap.

During the study block, participants were required to memorize the locations of each object within the environmental grid. The study block consisted of 48 trials (each of the 8 objects presented 6 times). A fixation cross appeared on the screen for $750 \mathrm{~ms}$, after which the spatial grid with landmarks was presented for 1,500 ms (interstimulus interval, ISI, of 2,250 ms). A test stimulus was presented after this time onto the grid and remained there for $2,000 \mathrm{~ms}$ or until a response was made. The presentations were pseudorandom (objects were presented randomly in a run of 8 , and this was repeated 6 times) so that numerous presentations of the same object did not coincide.

Participants in the test block were instructed to respond to objects in their correct location by clicking the left mouse button and by clicking the right mouse button when objects were presented out of location. The test block was split into two parts. Part 1 was a practice test with 8 trials. Four objects were presented in their correct position, and four were presented in an incorrect position. Presentations were randomized in the construction of the task on E-Prime, and this random order was the same for all participants. The participants were instructed to respond as speedily as possible within a 2-s time limit while also attempting to respond accurately. In Part 2, participants were required to recall the location of a particular stimulus that they had previously seen in the test block. There were 96 trials in total. The environment (including the landmarks) was rotated by $0^{\circ}$ (nonrotated), $90^{\circ}$ left, $90^{\circ}$ right, or $180^{\circ}$ (rotated) on each trial, with equal numbers of trials in each rotation. Objects were presented in either their correct or the incorrect location for each orientation (50:50). E-Prime logged reaction times and accuracy data for each participant. Reaction times were measured as the interval between presentation of the stimulus and the response and were recorded for both correct and incorrect trials. Failure to respond was classed as incorrect.

\section{Visual Paired-Associate (VPA) Task}

The Visual Paired-Associates Task (VPA; see Hogan et al., 2012) was designed to probe the 
status of episodic/associative memory. The task involved the presentation of a different local contextual background in conjunction to a stimulus pair. Stimuli pairs consisted of nonverbalizable achromatic abstract patterns. The task consisted of a study block containing 48 trials, followed by a test block containing 128 trials. The study block involved presenting the eight study pairs six times each in a pseudorandom order (objects were presented randomly in a run of 8 , which was repeated 6 times), such that consecutive presentations of the same object did not coincide. Each pair was associated with a unique constructed background.

Participants were required to indicate whether the two stimuli had been previously learned during the study phase (true-pair) or if the stimuli were recombined pairs (false-pair). Each pair continued to be presented along with a landscapemediated background; however, half of the pairs were presented on a congruent background (i.e., a background matching at least one element of the pair), whereas the other half were presented on an incongruent background (i.e., matching neither element of the pair). Consequently, there were four test conditions: true-congruent condition, true-incongruent condition, false-congruent condition, and false-incongruent condition. It was predicted that upon encoding of the stimulus pairs, participants would implicitly associate each pair with a contextual background and therefore would be more likely to remember a stimulus pair if it was presented along with its congruent local context.

\section{Statistics}

Data were initially collated in Excel and were subsequently graphed. A series of independent $t$ tests and mixed factorial analyses of variance (ANOVAs) with the appropriate post hoc tests (Tukey at the $5 \%$ level of significance) were employed. All statistics were carried out using the statistical package SPSS 19 for Windows (SPSS Software, Seattle, WA, USA). A star-based system of significance was also used to show the level of significance on our graphs: ${ }^{*} p<.05,{ }^{* *} p<.01$.

\section{Ethical approval and consent}

Ethical approval for this research was received by both the Research Ethics Committee of Tallaght Hospital and the National University of Ireland Maynooth Ethics Committee.

\section{RESULTS}

\section{Patient profiles}

Of the 16 patients, 12 people had suffered a stroke resulting from an infarct, 3 as a result of a hemorrhage, and 1 an undetermined presumed infarction. Four patients had infarcts located within the parietal region, 2 had middle cerebral artery infarcts, and 2 had infarcts in the internal capsule. Hemorrhages occurred in the parietal, temporal, and internal capsule of the three patients, respectively (see Table 1 for further details). All patients were tested with the MMSE and obtained an average score of $28 \pm 1.6$ out of a maximum score of 30 . The average length of stay was $20.37 \pm 5.1$ days.

\section{Paper-based tasks}

Comparison of the healthy controls $(36 \pm 2.6)$ to the patient group $(29 \pm 3.5)$ on the CFQ revealed no significant differences in absentmindedness between the groups, $t(39)=1.66, p>.05$. Likewise, scores on the NART showed no significant differences in estimated IQ (controls, $107 \pm 2.8$; patients, $110 \pm 3.8$ ); $t(39)=-0.733, p>.05$. Scores on the TMT (Trial B minus Trial A) also revealed no significant differences between the health control group $(59 \pm 0.3 \mathrm{~s})$ and the patient group (74 $\pm 11 \mathrm{~s}), t(39)=1.022, p>$ .05 , on cognitive flexibility.

The only paper-based task to show robust differences between the two groups was the RAVLT (see Figure 1). An initial $2 \times 8$ mixed factorial ANOVA was conducted with group (controls vs. patients) as the between-subjects factor and trial (1-8) as the within-subjects factor. Overall results indicated a significant main effect of trial, $F(7,266)=46.5$, $p<.001$, group, $F(1,38)=8.984, p<.01$, and Trial $\times$ Group interaction, $F(7,266)=4.32, p<$ .01 . Both groups significantly improved their word recall across the initial five trials, $F(4,152)=60.9$, $p<.01$, but the patient group had significantly lower scores overall, $F(1,38)=7.33, p<.01$; see Figure 1, A1-A5). In addition, while controls were able to distinguish, in general, between words that were presented from the A list and those presented on the interference list (List B; e.g., mean score on Trial A5 was $10.9 \pm 0.5$, which compares well to a mean score of $9.1 \pm 0.6$ on Trial A6), the patient group was more impaired, having a mean score of $9.0 \pm 0.7$ on Trial A5 and dropping to $5.5 \pm$ 0.9 on Trial A6. Indeed, an overall significant difference was found between the scores of controls and patients in Trials A6, $t(38)=3.328, p<.01$, and A7, $t(38)=3.338, p<.01$. 
TABLE 1

Breakdown of patient general characteristics, motor and language deficits, and affected brain area

\begin{tabular}{|c|c|c|c|c|c|c|}
\hline $\begin{array}{l}\text { Patient } \\
\text { number }\end{array}$ & Sex & Hand & $\begin{array}{c}\text { Education } \\
\text { level }^{a}\end{array}$ & $\begin{array}{l}\text { Interlocking } \\
\text { Finger Test } \\
(\text { out of } 4)^{b}\end{array}$ & Motor/language deficits & Injury type/area affected \\
\hline 1 & M & $\mathrm{R}$ & 3 & 4 & $\begin{array}{l}\text { (L) Hemiparesis/ dysarthria: } 78 \% \\
\text { word, } 84 \% \text { sentence intelligibility }\end{array}$ & Infarct/ (L) parietal \\
\hline 2 & $\mathrm{~F}$ & $\mathrm{R}$ & 2 & 4 & Expressive dysphasia & Infarct/ (R) posterior parietal \\
\hline 3 & M & $\mathrm{R}$ & 5 & 4 & (L) sided weakness & $\begin{array}{l}\text { Ischemia, intracranial } \\
\text { haemorrhage/ }(\mathrm{R}) \text { basal ganglia }\end{array}$ \\
\hline 4 & M & $\mathrm{R}$ & 3 & 4 & (R) Facial droop/ dysarthria & $\begin{array}{l}\text { Hemorrhagic infarct/ (L) internal } \\
\text { capsule and thalamus }\end{array}$ \\
\hline 5 & M & $\mathrm{R}$ & 3 & 4 & $\begin{array}{l}\text { Mild dysphagia/ word finding } \\
\text { problems }\end{array}$ & Infarct/ (L) middle cerebral artery \\
\hline 6 & M & $\mathrm{R}$ & 3 & - & $\begin{array}{l}\text { (L) sided weakness/ dysarthria: } \\
78 \% \text { word, } 84 \% \text { language } \\
\text { intelligibility }\end{array}$ & $\begin{array}{l}\text { Lacunar infarcts scattered } \\
\text { throughout the brain, }(\mathrm{R}) \\
\text { internal capsule infarct } \\
\text { calcification }(\mathrm{L}) \text { cerebellum }\end{array}$ \\
\hline 7 & $\mathrm{~F}$ & $\mathrm{R}$ & 1 & 4 & Slight hemiparesis & Infarct/ (R) middle cerebral artery \\
\hline 8 & $\mathrm{~F}$ & $\mathrm{R}$ & 1 & 3 & Slight hemiparesis/ slight aphasia & Infarct/ $(\mathrm{R})$ posterior parietal \\
\hline 9 & M & $\mathrm{R}$ & 2 & 4 & (L) sided facial droop & $\begin{array}{l}\text { Chronic nonspecific ischemia/ } \\
\text { multiple regions }\end{array}$ \\
\hline 10 & M & $\mathrm{R}$ & 2 & - & $\begin{array}{l}\text { (R) sided facial weakness/ } \\
\text { dysarthria }\end{array}$ & Hemorrhage/ subcortical parietal \\
\hline 11 & M & $\mathrm{R}$ & 2 & 4 & Dysphasia & Infarct/ (L) internal capsule \\
\hline 12 & M & $\mathrm{R}$ & 1 & 4 & (R) Hemiparesis & $\begin{array}{l}\text { Infarct/ }(\mathrm{L}) \text { corona radiate and }(\mathrm{R}) \\
\text { frontal }\end{array}$ \\
\hline 13 & $\mathrm{~F}$ & $\mathrm{R} \& \mathrm{~L}$ & 2 & 4 & $\begin{array}{l}\text { Expressive dysphasia, alexia, } \\
\text { anomia }\end{array}$ & $\begin{array}{l}\text { Hemorrhage/ }(\mathrm{L}) \\
\text { parietal-temporal }\end{array}$ \\
\hline 14 & M & $\mathrm{R}$ & 1 & 4 & Receptive and expressive dysphasia & Infarct/ (L) parietal-occipital \\
\hline 15 & M & $\mathrm{L}$ & 1 & 4 & - & Infarct/ (L) frontal-parietal \\
\hline 16 & $\mathrm{~F}$ & $\mathrm{R}$ & 1 & - & - & Suspected infarct/ (R) parietal lobe \\
\hline
\end{tabular}

Note. $\mathrm{M}=$ male. $\mathrm{F}=$ female. $\mathrm{R}=$ right. $\mathrm{L}=$ left.

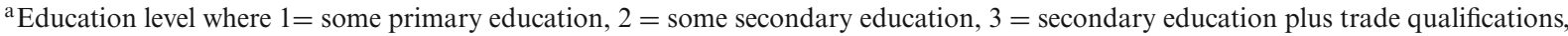
$4=$ secondary school completed, and $5=$ some tertiary education (see Willshire et al. 1991). ${ }^{\mathrm{b}}$ The ability to complete four different types of two-handed interlocking movements (loops, piggies, butterfly, and Vulcan) with a maximum score of 4 (see Moo et al., 2003, for details).

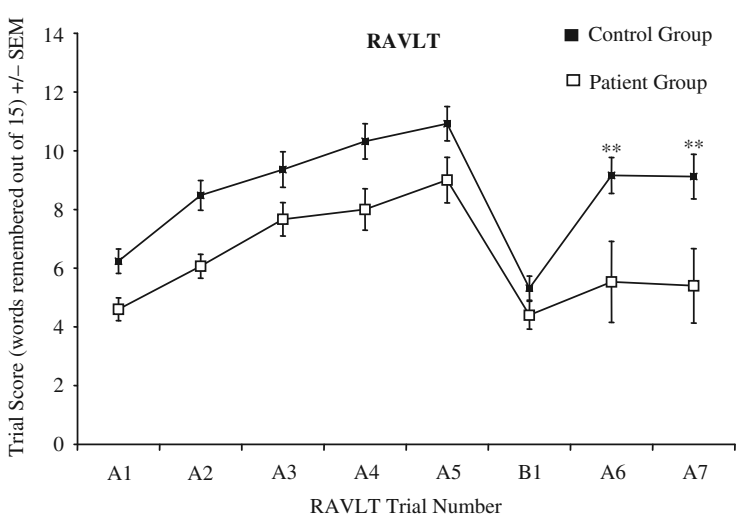

Figure 1. A line graph depicting control and patients score on the RAVLT (Rey Auditory Verbal Learning Task) across Trials 1-5, the interference Trial B, and the delayed Trials A6 and A7.

\section{Computer-based tasks}

We initially compared the two groups on the visual search task. The control group was significantly more accurate $(87 \pm 0.5 \%)$ in identifying when the target was present in the array of stimuli than was the patient group $(73 \pm 5.5 \%), t(29)=2.517$, $p<.05$ (see Figure 2); however, when the target was absent, there was no difference between the groups,

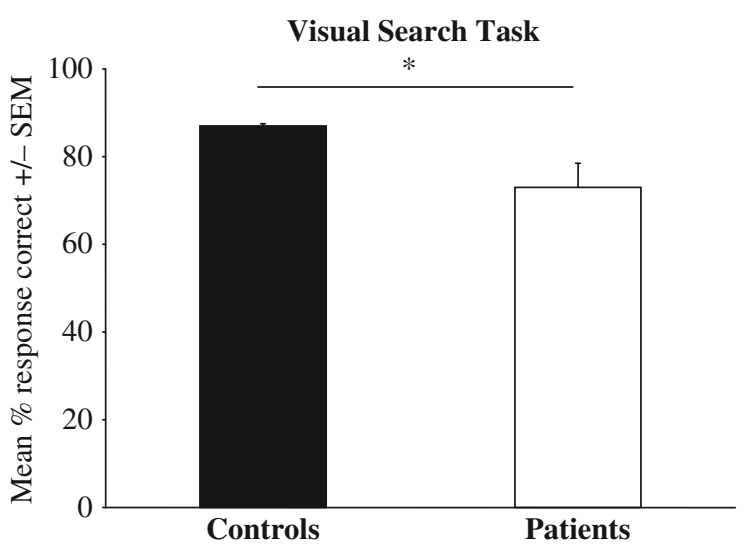

Figure 2. Bar chart comparing the overall accuracy of control and patients on the visual search task (for target present). 
$t(29)=0.565, p>.05$. We also compared the time taken for both groups to identify whether the target was present or absent. When the target was present, no significant difference between the groups was noted, $F(1,27)=1.704, p>.05$; nor was there an overall reaction time difference between correct and incorrect responses (accuracy), $F(1,27)=0.993$, $p>.05$, and no interaction effect, $F(1,27)=0.001$, $p>.05$, was observed. However, when the target was absent, no overall significant main effect of accuracy was reported, $F(1,15)=1.584, p>.05$, and neither group nor the interaction of Group $\times$ Accuracy proved significant, $F(1,15)=0.731, p>$ .05 , and $F(1,15)=0.554, p>.05$, respectively.

We then compared the two groups on the Visual Paired-Associates Task. Figure 3 compares the accuracy for both control and patient groups in four different conditions. The first condition (true-pair congruent) was when the stimuli were in the correct pairing, and this pairing was also matched to the background that had been presented during the study phase. The second condition (true-pair incongruent) was when the stimuli were presented in their correct pairing but the background was not matched to that presented during the study block (note that participants only had to respond to whether the stimulus pair was correct or not). The third condition (false-pair congruent) was when the stimulus pair was not matched but the background was correct for one element of the pair presented. The final condition (false-pair incongruent) was when the stimuli were not correctly paired, and the background was also not matched to either element. A $2 \times 4$ mixed factorial ANOVA using group (control vs. patient) as the between-subjects measure
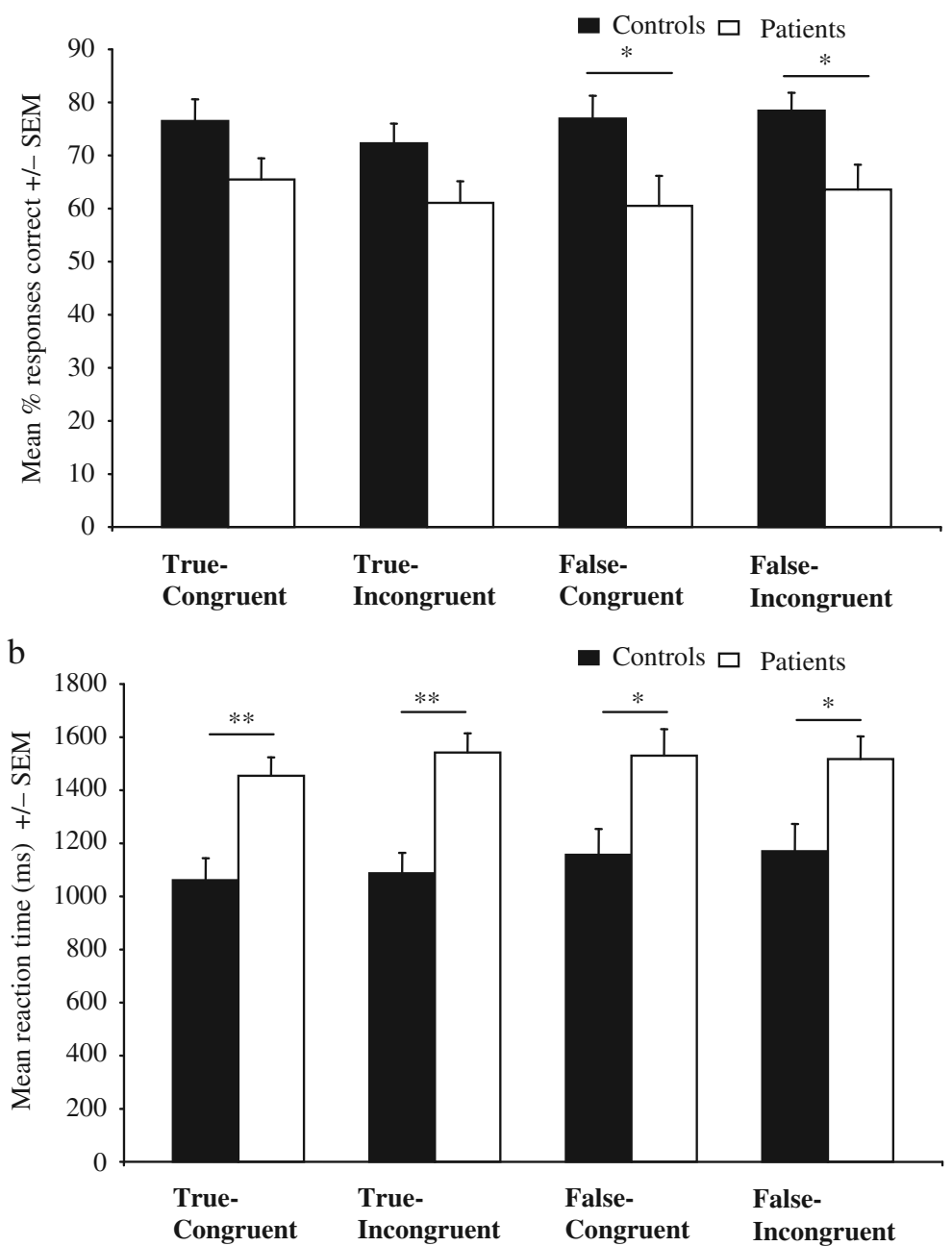

Figure 3. Bar chart comparing the accuracy (a) and reaction time (b) of the control group and patient group on the Visual PairedAssociates Task for all four experimental conditions (true-congruent, true-incongruent, false-congruent, and false-incongruent) for correct response. ${ }^{*} p<.05,{ }^{* *} p<.01$. 
and condition (true-congruent, true-incongruent, false-congruent, and false-incongruent) as the within-subjects measure was conducted. No overall significant effect was found for condition, $F(3$, $108)=1.072, p>.05$, or Condition $\times$ Group interaction, $F(3,108)=0.455, p>.05$, but an overall effect for group was found, $F(1,36)=6.764$, $p<.05$ (see Figure 3a), with the controls making significantly more accurate responses than the patient group for the two false conditions: $t(36)=$ 2.327, $p<.05$, and $t(36)=2.595, p<.05$, for false-congruent and false-incongruent conditions, respectively.

Similar analyses were conducted for reaction time for correct responses made in each condition (Figure 3b). Overall, similar to the accuracy results, no significant difference was found for condition, $F(3,108)=2.222, p>.05$, or Condition $\times$ Group interaction, $F(3,108)=0.710, p>.05$, but an overall effect of group was found, $F(1,36)=9.620$, $p<.01$, with the controls making significantly faster responses than the patient group for all four conditions: $t(36)=3.233, p<.01 ; t(36)=3.955$, $p<.01 ; t(36)=2.504, p<.05$; and $t(36)=2.312$, $p<.05$, for the true-congruent, true-incongruent, false-congruent, and false-incongruent conditions, respectively.

The final computer-based task employed was the Spatial Grid Task. Figure 4a demonstrates that the controls were very accurate in recalling an object's location, obtaining a mean correct score of $83 \pm 2.4 \%$; however, this accuracy decreased to $54 \pm 2.9 \%$ when recalling an object's location from an alternative viewpoint (a viewpoint that was not presented during the study block). Similarly, the patient group was relatively accurate in their attempts to recall an object's location, obtaining a mean accuracy of $66.5 \pm 5.1 \%$; however, like the control group, their accuracy also decreased to $41 \pm 4 \%$ when recalling the object's location from a rotated viewpoint. A $2 \times 2$ mixed factorial ANOVA confirmed overall significant effects of view (nonrotated vs. rotated), $F(1,34)=112.58$, $p<.001$, and of group (control vs. patients), $F(1$, $34)=12.59, p<.001$, but no significant View $\times$ Group interaction effect, $F(1,34)=0.901, p>$ .05 . Two independent $t$ tests showed a significant difference between the control and the patient

a

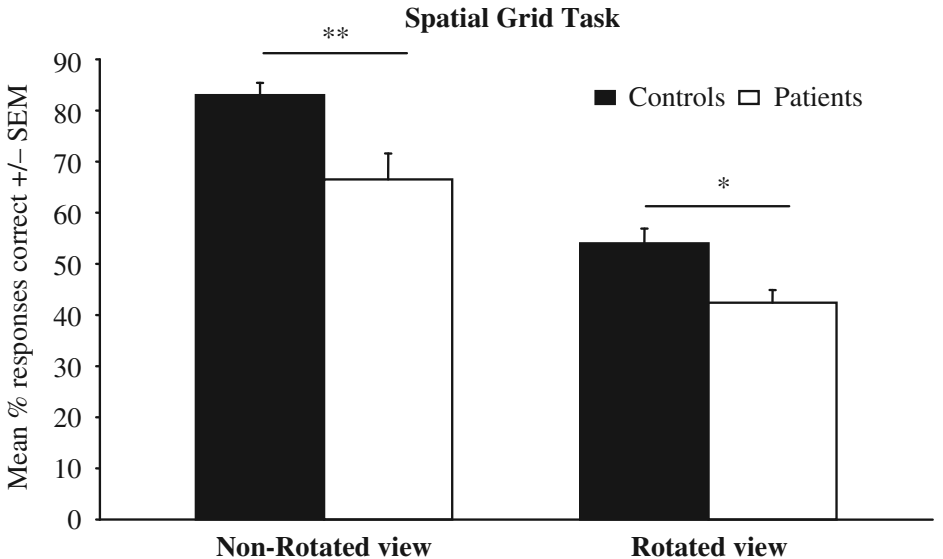

$\mathrm{b}$

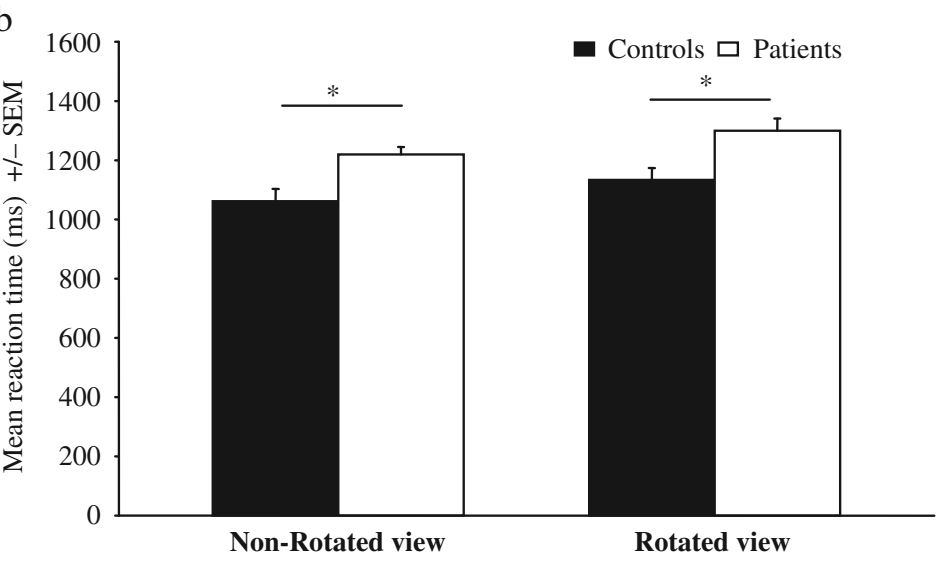

Figure 4. Bar chart comparing the accuracy (a) and reaction time (b) of both the control group and patient group on the Spatial Grid Task for correct responses. ${ }^{*} p<.05,{ }^{* *} p<.01$. 
group for both the nonrotated and the rotated condition, $t(34)=3.296, p<.01$, and $t(34)=2.662$, $p<.05$, respectively. Examination of the reaction times (Figure 4b) reflected the accuracy data, with the control group making accurate responses significantly quicker, irrespective of viewpoint, than the patient group, $F(1,34)=10.257, p<.01$, with responses overall on the nonrotated condition being quicker than those in the rotated conditions, $F(1,34)=5.19, p<.05$. There was no View $\times$ Group interaction effect, $F(1,34)=0.15, p>$ .05. Two independent $t$ tests showed a significant difference between the control and the patient group for both the nonrotated and the rotated conditions, $t(34)=2.66, p<.05$, and $t(34)=2.67$, $p<.05$, respectively.

\section{DISCUSSION}

Here we describe differences between stroke survivors and healthy controls on a number of different cognitive tasks. Specifically, we report some attentional deficits and robust memory impairments. The memory impairments observed can be broken down into deficits in verbal working memory, visual associative memory, and spatial object location memory. Such a pattern of deficits was observed despite patients and controls having similar scores on the more generalized tests of cognitive impairment and intelligence such as the CFQ and NART (as well as patients obtaining a score on the MMSE of mean of 28/30). As some of these tests (e.g., MMSE) are used regularly in a clinical situation to assess cognitive function, our findings suggest that it is important to widen the range of cognitive assessments in order to detect subtle impairments that may be brought about by stroke, and, once a deficit is detected, it is important to follow up with more in-depth domain-specific assessments to gain a complete profile of cognitive ability post stroke. Indeed, our findings add to the growing number of concerns that have been raised regarding the adequacy of the MMSE as a sole assessment for cognitive function. Some argue that it is biased toward verbal items and fails to adequately measure functions such as attending to relevant input, abstract problem solving, long-term retrieval, and visuospatial ability (Strauss et al., 2006). Further, these authors argue that disturbances of the right hemisphere (common in stroke) are neglected, and that mild impairments may go undetected. Recall and attention MMSE subtests tend to be the least reliable (Olin \& Zelinski, 1991), with low retest correlations reported. In addition, other commentators (e.g., Giordani et al., 1990) state that the
MMSE subscales and individual items should not be viewed as measures of specific aspects of cognition, as the set of cognitive domains sampled by the subscales may be less than the seven categories into which the items are grouped (Tombaugh \& McIntyre, 1992); as such, they should not be used in lieu of a comprehensive assessment of cognitive function. In conclusion, suspicions of the presence of impairment based on failures on one or more of the MMSE items needs to be followed up by in-depth assessment of cognitive functions, as the presence of cognitive deficits should not be diagnosed on the basis of MMSE scores alone (Strauss et al., 2006).

Attention deficits are regularly observed following stroke, with a prevalence rate of between $31 \%$ and $35 \%$ and up to $60 \%$ in some cases (BarkerCollo, Feigin, Lawes, Senior, \& Parag, 2010 Hyndman, Pickering, \& Ashburn, 2008). Although such deficits do improve soon after stroke, they may still be present at least 6 months later (Stapleton, Ashburn, \& Stack, 2001). It is important that such attentional deficits are recognized early as better attentional performance is often associated with better functional outcome-for example, for motor abilities, and so on (McDowd, Filion, Pohl, Richards, \& Stiers, 2003). Although the TMT is often used to assess attentional difficulties as well as executive function, we failed to see any difference between the control and the patient groups on this measure. However, our visual search task revealed significant differences, with the patient group failing to identify the target when it was present among a number of distractor stimuli. We believe that the impairments observed were the result of a visual attention deficit rather than a visual problem per se, as all patients had good vision, and patients with severe spatial neglect were excluded from the study. Our findings confirm a recent study by Erez, Katz, Ring, and Soroker (2009), who reported that their computerized visual search tasks can clearly differentiate not only between stroke patients and controls but also between different patient groups, such as those who suffer from unilateral spatial neglect and those who do not. While our visual search task was limited (conjunction search only), it can easily be adapted and modified so that it can test for spatial neglect or incorporate a feature search test. Such approaches may be more useful and more sensitive than standard paper-and-pencil tests (line cancellation, etc.), as computerized tasks are less likely to be biased by learning effects (Erez et al., 2009).

Our study also identified profound memory difficulties in patients recovering from stroke that include deficits in verbal recall (as 
observed in the RAVLT), associative memory (Visual Paired-Associates Task), and spatial processing/location memory (Spatial Grid Task). These deficits were observed despite finding no differences in the NART and CFQ, some of which contain a memory component. While the RAVLT has been used with patients suffering from Alzheimer's disease (e.g., Gleichgerrcht, Torralva, Martinez, Roca, \& Manes, 2011), there has been limited use of the RAVLT with stroke patients. One recent study (Schoenberg et al., 2006), however, does describe impairments in a number of patient groups, including a stroke group, on the RAVLT when compared to metanorms for healthy participants. Further, the scores reported in their study compare favorably with those observed here, suggesting that this easy-to-administer task may be useful as part of a larger battery of tests assessing memory. Results from the VPA task indicate that patients not only have a general impairment in recalling correct associations, but they had difficulties with specific aspects of the task. For example, although the patient group was as accurate as the control group in identifying the correct pair, they were significantly slower in making this choice. More specific deficits were observed when they had to identify a false (recombined) stimulus pair. Patients were both less accurate and slower in their response (irrespective of the background) than were the control group. While (similar to the RAVLT) modified versions of this task have been used to examine cognition in patients with Alzheimer's disease (Oda, Yamamoto, \& Maeda, 2009), the VPA is seldom used with stroke patients.

While the Spatial Grid Task (Murphy et al., 2009) was originally designed in our laboratory to explore the ability of healthy participants to recall an object's location from a studied viewpoint (egocentric) compared to a nonstudied rotated view (allocentric), the findings that stroke patients are impaired in both nonrotated and rotated conditions suggest that this task may be sensitive to assessing subtle spatial processing difficulties. While spatial deficits are commonly observed in stroke patients, with damage centered on the parietal region (van Asselen et al., 2009), this task may prove useful as a means of probing relational memory problems as well as spatial processing difficulties. Based on a modified version of the task used by Johnsrude et al. (1999) in their examination of the hippocampal patient Jon, it may also be possible to use this task to distinguish between parietal patients and nonparietal (e.g., hippocampal and temporal) patients. Unfortunately, low patient numbers in our study did not allow us to make such comparisons. As in Murphy et al. (2009), the task can easily be modified and may be used to check implicit or long-term spatial memory. We suggest that the Spatial Grid Task along with the RAVLT and VPA could become part of a more comprehensive assessment of memory in stroke patients during recovery.

There are a number of limitations with our study. Stroke patients are, by their nature, a heterogeneous group, and therefore it is very difficult to ascertain and correlate the region of brain damage to the type of cognitive deficit. In addition, our sample size is quite small, but we purposely excluded arm weakness, expressive aphasia, and the presence of spatial neglect in order that these comorbid problems would not adversely affect the scores. Although many of the tests used (e.g., the RAVLT and NART) are sensitive to education and age, we used controls that were age and educated matched to the patient group and did not rely on metanorms for healthy participants. Furthermore, while the majority of people did suffer from dysarthria to various degrees (see Table 1), this did not seem to affect their scores on the NART (as no significant difference was found between the patients and controls on this test).

In conclusion, we believe that there should be a standardized multidomain cognitive assessment for stroke survivors (e.g., McDonnell et al., 2011), and we propose that once a cognitive deficit is identified, there should be a further in-depth, domain-specific assessment of the particular cognitive impairment. Our tasks have been shown to be sensitive to assessing different aspects of both attention and memory in stroke patients and may therefore form part of this in-depth assessment within these specific domains.

Original manuscript received 19 March 2011 Revised manuscript accepted 2 April 2012 First published online 31 May 2012

\section{REFERENCES}

Barker-Collo, S., Feigin, V., Lawes, C., Senior, H., \& Parag, V. (2010). Natural history of attention deficits and their influence on functional recovery from acute stages to 6 months after stroke. Neuroepidemiology, 35(4), 255-262.

Benton, A. L. (1990). Constructional apraxia. In F. Boller \& J. Grafman (Eds.), Handbook of neuropsychology (Vol. 2). Amsterdam, The Netherlands: Elsevier.

Broadbent, D. E., Cooper, P. F., FitzGerald, P., \& Parkes, K. R. (1982). The Cognitive Failures Questionnaire (CFQ) and its correlates. British Journal of Clinical Psychology, 21, 1-16.

Cicerone, K. D., Dahlberg, C., Malec, J. F., Langenbahn, D. M., Felicetti, T., Kneipp, S., et al. (2005) Evidence-based cognitive rehabilitation: Updated review of the literature from 1998 through 2002 
Archives of Physical Medicine and Rehabilitation, 86(8), 1681-1692.

Erez, A. B., Katz, N., Ring, H., \& Soroker, N. (2009). Assessment of spatial neglect using computerised feature and conjunction visual search tasks. Neuropsychological Rehabilitation, 19(5), 677-695.

Farrell, M. J., \& Robertson, I. H. (2000). The automatic updating of egocentric spatial relationships and its impairment due to right posterior cortical lesions. Neuropsychologia, 38(5), 585-595.

Folstein, M. F., Folstein, S. E., \& McHugh, P. R. (1975). "Mini-mental state." A practical method for grading the cognitive state of patients for the clinician. Journal of Psychiatric Research, 12(3), 189-198.

Giordani, B., Boivin, M. J., Hall, A. L., Foster, N. L., Lehtinen, S. J., Bluemlein, L. A., et al. (1990). The utility and generality of Mini-Mental State Examination scores in Alzheimer's disease. Neurology, 40(12), 1894-1896.

Gleichgerrcht, E., Torralva, T., Martinez, D., Roca, M., \& Manes, F. (2011). Impact of executive dysfunction on verbal memory performance in patients with Alzheimer's disease. Journal of Alzheimer's Disease, 23(1), 79-85.

Hofgren, C., Bjorkdahl, A., Esbjornsson, E., \& Sunnerhagen, K. S. (2007). Recovery after stroke: Cognition, ADL function and return to work. Acta Neurologica Scandinavica, 115, 73-80.

Hogan, M. J., Kenney, J. P. M., Keane, M. A., Roche, R. A. P., Moore, J. L., Kaiser, J., et al. (2012). Behavioural and electrophysiological effects of visual paired associate context manipulations during encoding and recognition in younger adults, older adults and older cognitively declined adults. Experimental Brain Research, 216(4), 621-633.

Hommel, M., Miguel, S. T., Naegele, B., Gonnet, N., \& Jaillard, A. (2009). Cognitive determinants of social functioning after a first ever mild to moderate stroke at vocational age. Journal of Neurology, Neurosurgery, and Psychiatry, 80, 876-880.

Humphreys, G. W., Allen, H. A., \& Mavritsaki, E. (2009). Using biologically plausible neural models to specify the functional and neural mechanisms of visual search. Progress in Brain Research, 176, 135-148.

Hyndman, D., Pickering, R. M., \& Ashburn, A. (2008). The influence of attention deficits on functional recovery post stroke during the first 12 months after discharge from hospital. Journal of Neurology, Neurosurgery, and Psychiatry, 79, 656-663.

Irish Heart Foundation Council on Stroke. (2001). Towards excellence in stroke care. Dublin, Ireland: Irish Heart Foundation.

Irish Heart Foundation National Audit of Stroke Care. (2008). Dublin, Ireland: Irish Heart Foundation.

Johnsrude, I. S., Owen, A. M., Crane, J., Milner, B., \& Evans, A. C. (1999). A cognitive activation study of memory for spatial relationships. Neuropsychologia, 37, 829-841.

Keith, R. A., Granger, C. V., Hamilton, B. B., \& Sherwin, F. S. (1987). The Functional Independence Measure: A new tool for rehabilitation. In M. G. Eisenberg \& R. C. Grzesiak (Eds.), Advances in clinical rehabilitation (Vol. 1, pp. 6-18. New York, NY: Springer-Verlag.

Mahoney, F. I., \& Barthel, D. W. (1965). Functional evaluation-The Barthel Index. Maryland State Medical Journal, 14, 61-65.
Mathuranath, P. S., Nestor, P. J., Berrios, G. E., Rakowicz, W., \& Hodges, J. R. (2000). A brief cognitive test battery to differentiate Alzheimer's disease and frontotemporal dementia. Neurology, 55(11), 1613-1620.

McDonnell, M. N., Bryan, J., Smith, A. E., \& Esterman, A. J. (2011). Assessing cognitive impairment following stroke. Journal of Clinical and Experimental Neuropsychology, 3, 1-9.

McDowd, J., Filion, D. L., Pohl, P. S., Richards, L. G., \& Stiers, W. (2003). Attentional abilities and functional outcomes following stroke. Journal of Gerontology: Psychological Sciences, 58, 45-53.

Mesulam, M. M. (1981). A cortical network for directed attention and unilateral neglect. Annals of Neurology, 10, 309-325.

Moo, L. R., Slotnick, S. D., Tesoro, M. A., Zee, D. S., \& Hart, J. (2003). Interlocking finger test: A bedside screen for parietal lobe dysfunction. Journal of Neurology, Neurosurgery, and Psychiatry, 74(4), 530-532.

Murphy, J. S., Wynne, C. E., O’Rourke, E. M., Commins, S., \& Roche, R A. (2009). High-resolution ERP mapping of cortical activation related to implicit object-location memory. Biological Psychology, 82(3), 234-245.

Nelson, H. E. (1982). National Adult Reading Test (NART), test manual. Windsor, UK: NFER Nelson.

Oda, H., Yamamoto, Y., \& Maeda, K. (2009). The neuropsychological profile in dementia with Lewy bodies and Alzheimer's disease. International Journal of Geriatric Psychiatry, 24(2), 125-131.

Olin, J. T., \& Zelinski, E. M. (1991). The 12-month reliability of the Mini Mental State Examination. Psychological Assessment, 3, 427-432.

Patel, M., Coshall, C., Rudd, A. G., \& Wolfe, C. D. (2003). Natural history of cognitive impairment after stroke and factors associated with its recovery. Clinical Rehabilitation, 17(2), 158-166.

Pattie, A. H. (1981). A survey version of the Clifton Assessment Procedure for the Elderly (CAPE). British Journal of Clinical Psychology, 209(3), 173-178.

Pendlebury, S. T., \& Rothwell, P. M. (2009). Prevalence, incidence, and factors associated with pre-stroke and post-stroke dementia: A systematic review and meta-analysis. Lancet Neurology, 8(11), 1006-1018.

Reitan, R. M. (1992). Trail Making Test. Manual for administration and scoring. South Tucson, AZ: Reitan Neuropsychology Laboratory.

Rey, A. (1941). L'examen psychologique dans les cas d'encephalopathie traumatique [Psychological examination of traumatic encephalopathy]. Archives of Psychology, 28, 286-340.

Schoenberg, M. R., Dawson, K. A., Duff, K., Patton, D., Scott, J. G., \& Adams, R. L. (2006). Test performance and classification statistics for the Rey Auditory Verbal Learning Test in selected clinical samples. Archives of Clinical Neuropsychology, 21(7), 693-703.

Stapleton, T., Ashburn, A., \& Stack, E. (2001). A pilot study of attention deficits, balance control and falls in the subacute stage following stroke. Clinical Rehabilitation, 15, 437-444.

Strauss, E., Sherman, E. M. S., \& Spreen, O. (2006). A compendium of neuropsychological tests: 
Administration, norms, and commentary (3rd ed.). New York, NY. Oxford University Press.

Tombaugh, T. N., \& McIntyre, N. J. (1992). The MiniMental State Examination: A comprehensive review. Journal of the American Geriatrics Society, 40(9), 922-935.

Treisman, A. M., \& Gelade, G. (1980). A featureintegration theory of attention. Cognitive Psychology, 1(1), 97-136.

Van Asselen, M., Kessels, R. P., Frijns, C. J., Kappelle, L. J., Neggers, S. F., \& Postma, A. (2009). Object- location memory: A lesion-behavior mapping study in stroke patients. Brain and Cognition, 71(3), 287-294.

Warrington, E. K., \& Taylor, A. M. (1973). The contribution of the right parietal lobe to object recognition. Cortex, 9, 152-164.

Willshire, D., Kinsella, G., \& Prior, M. (1991). Estimating WAIS-R IQ from the National Adult Reading Test: A cross-validation. Journal of Clinical and Experimental Neuropsychology, 13(2), 204-216. 